\title{
P-S Standard
}

\author{
Egger Mielberg \\ egger.mielberg@gmail.com
}

17.02.2019

\begin{abstract}
.
An information of any kind can take two forms, at least. The first one is interpreted as a solution to a problem. The second one is interpreted as a plain text. For the last several centuries the mankind generated tons of different information relating to a number of different fields. Number of standards were created for the purpose of ruling its applications. But what percent of the generated information is a real solution for a real problem? $10 \%$ or less?

We propose a simple solution for such an important problem as a "converting text to solution", IPSC standard. It is abbreviation for "Introduction-Problem-Solution-Conclusion".
\end{abstract}

\section{Introduction}

A single solution (method, algorithm, etc.) can be applicable to one or a set of problems. As many problems of one business or scientific field can be related to each other, it is extremely important to be not diverted from the shortest way in the process of finding the solution.

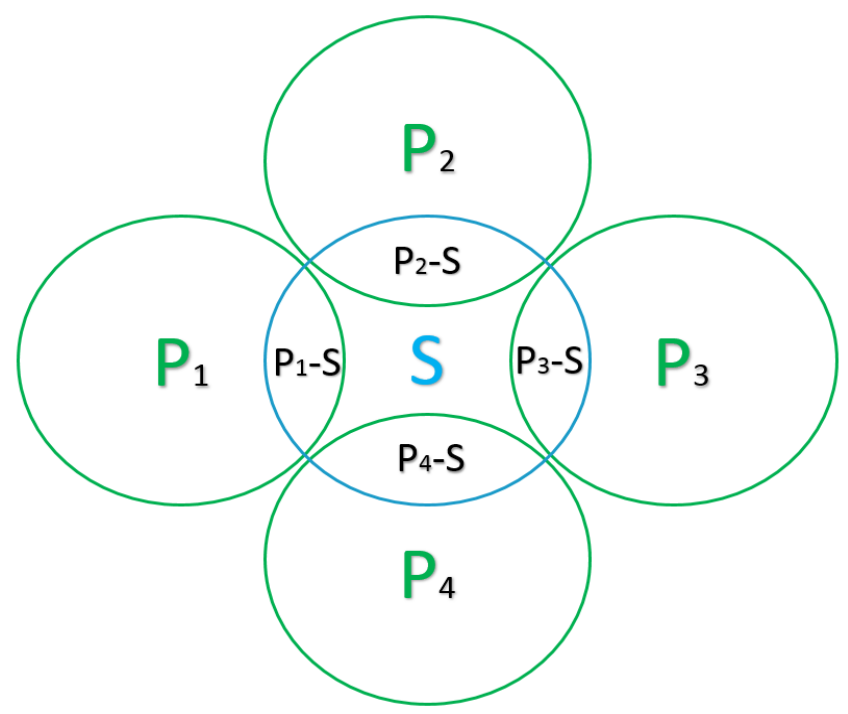


The number of problems $\left(\mathrm{P}_{\mathrm{N}}\right)$ can be substantially bigger then four. As a results, the set of " $\mathrm{P}_{\mathrm{N}}-\mathrm{S}$ " subsets can increase exponentially.

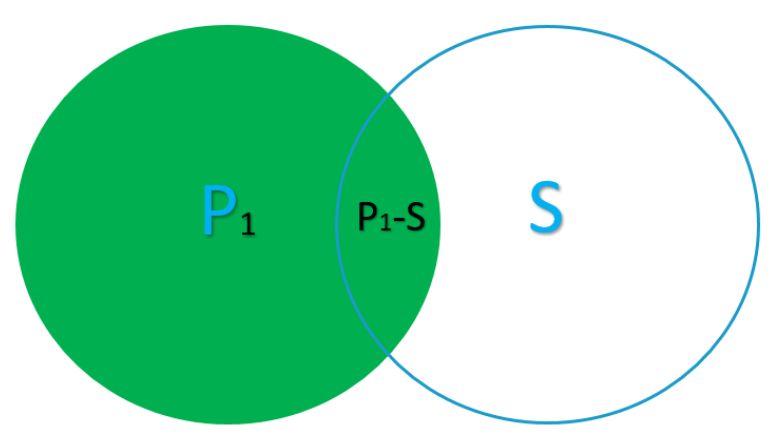

A search pattern [3] that can directly lead to " $P_{1}-S$ " subset is the best one for $\mathrm{P}_{1}$ problem.

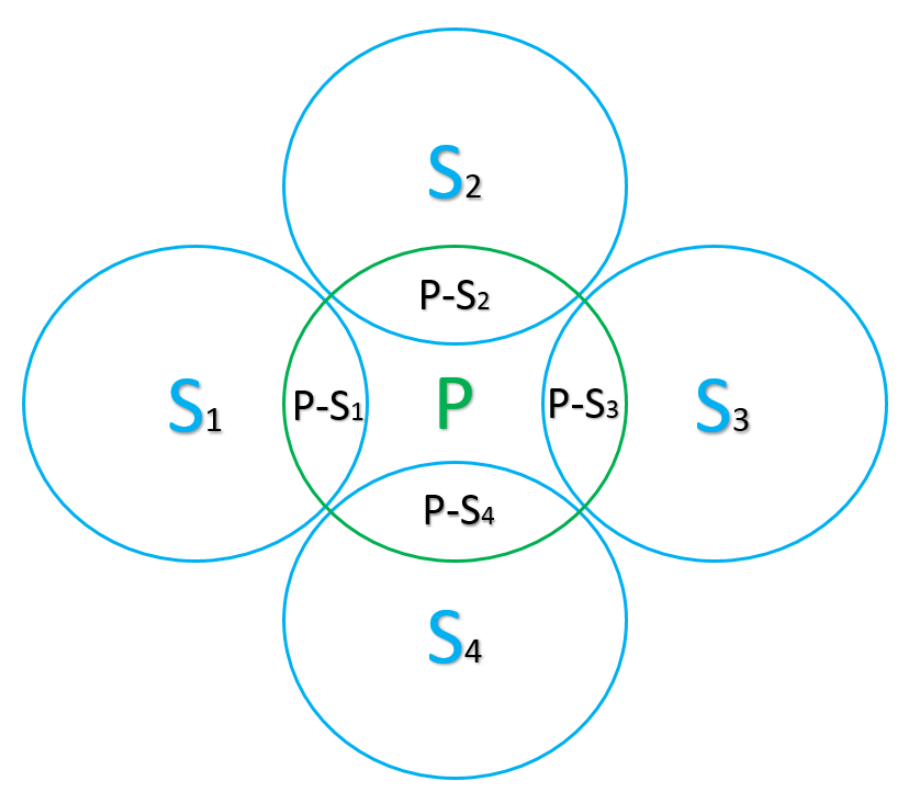

In case of existence of more than one solution for a single problem, the pattern can be found in substantially less time.

In terms of neurobiology, the process of searching solution is extremely complicated. Each neuron can have over 20 thousands of connections (synapses) to other neurons. Now, imagine that approximate number of all neurons is about 100 billion in a one single humankind head. The next step which could be proposed is to imagine the number of all possible connections between all the neurons. But we stop here. 
Let us go to the example. We have a single task and want to find a single solution for it. Given rows of numbers each of which is located in different field of our brain. Each number of the row has own unique knowledge and history of its formation (life experience). Each row consists of a set of the numbers which form the row by a single sense. The row was chosen for clearance sake. In reality, the set of numbers can form a zig-zag structure.

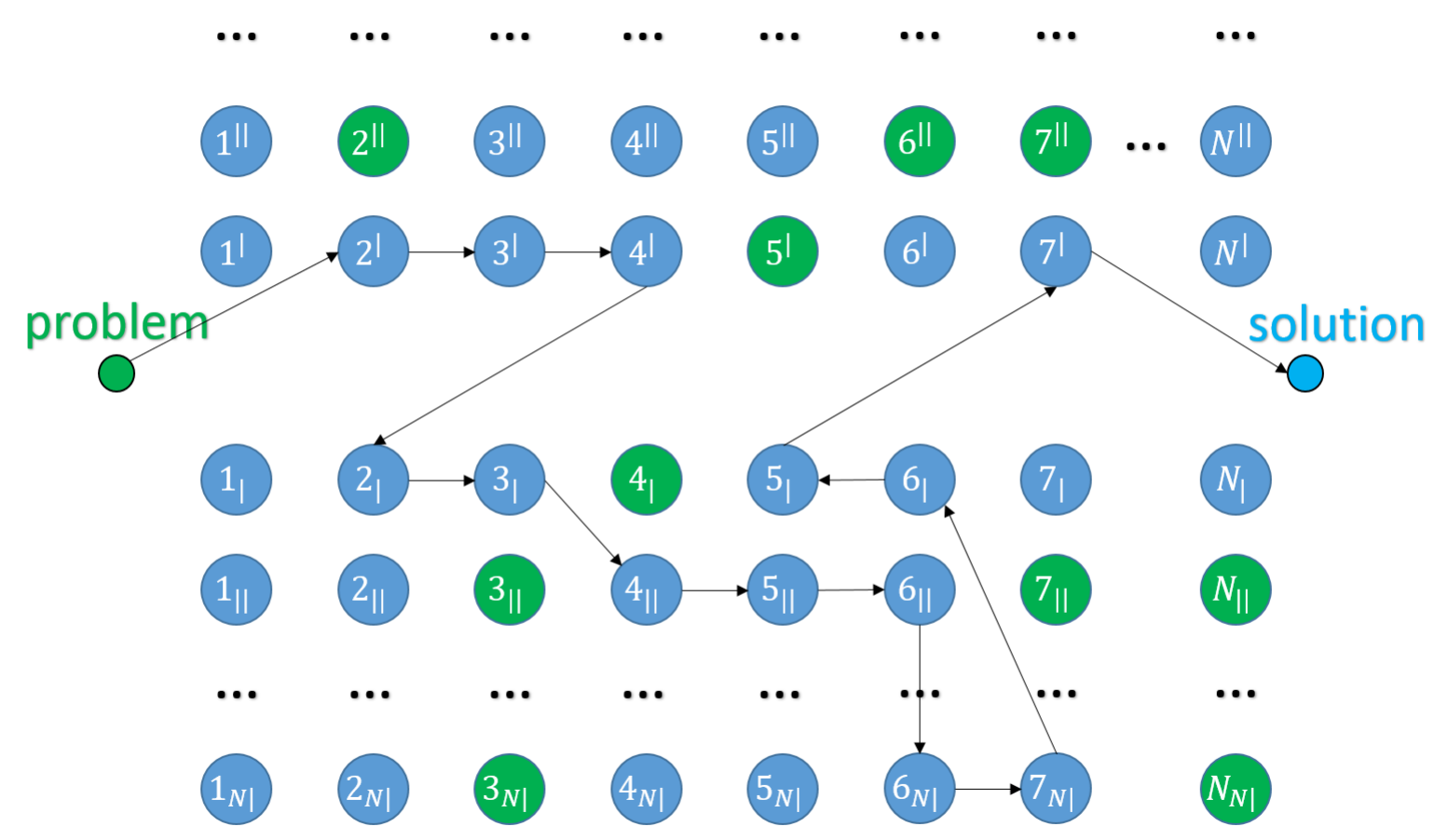

As we see on the figure above, the search pattern for a single solution can be extremely complex and exhausting. The shown search pattern is pretty good yet as it did not encounter other task on its way to the solution.

Obviously, the green-colored numbers (knowledge) can only have input channels from another ones as they still do not have any solution.

In reality, a scheme of connections of the numbers looks much more redoubtable. For example, it might look like this: 


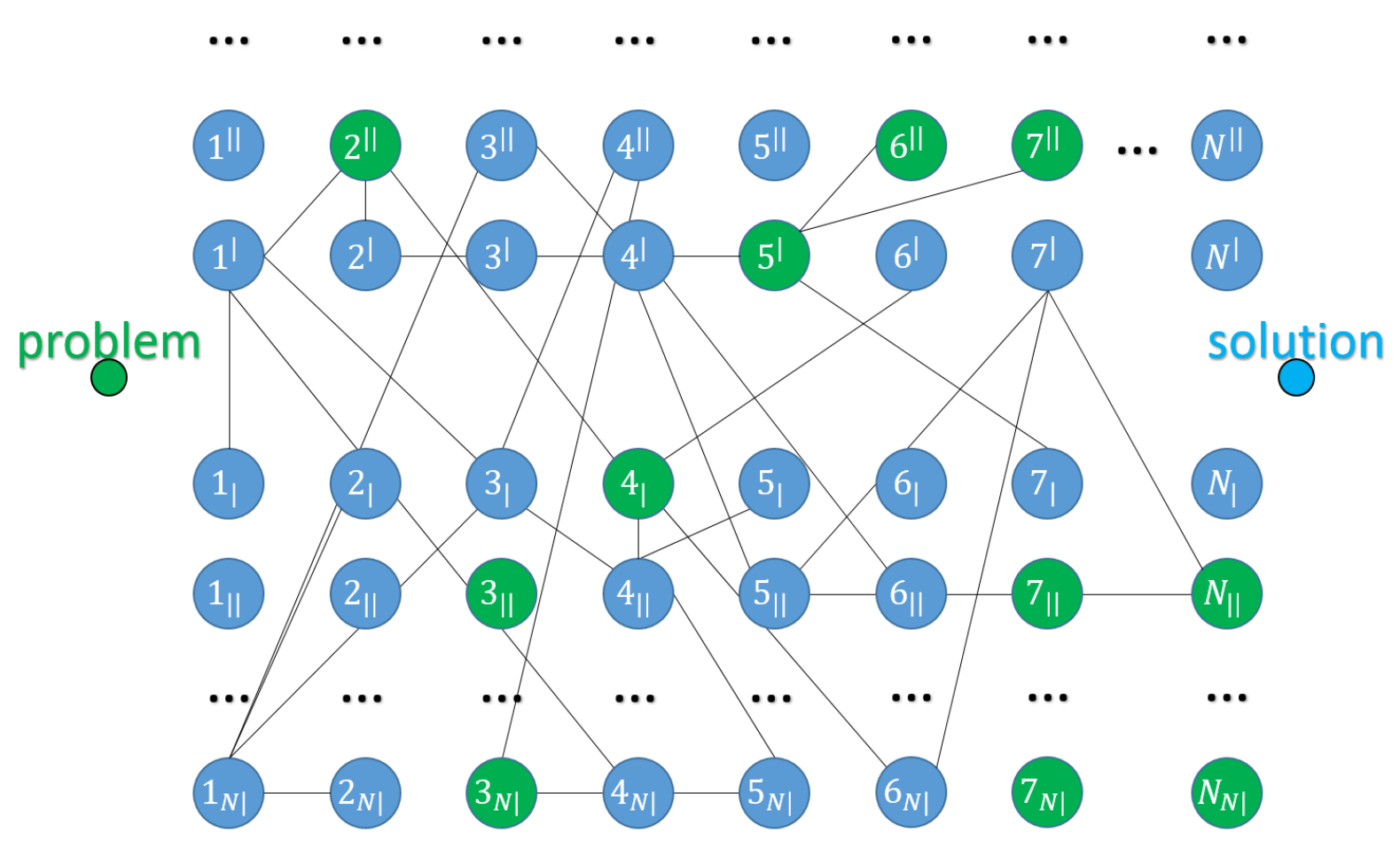

As we see on the figure above, only a single green-colored number on the way to the solution can lead to years of hard work for finding an alternative way. That is why it is extremely important to be capable of finding a shortest-way-search-pattern for the problem of any kind.

In this article we describe a simple idea of how the pattern can be found with less efforts.

\section{Problem}

The problem which is described in this article mainly concerns documents in different fields that declare about some innovations or problems they are used to solve.

Let us have a look at a conventional template of such document.

Content

1. Introduction

2. Section 1 heading

2.1. Subsection heading

2.2. Subsection heading

2.3.

3. Section 2 heading

3.1. Subsection heading

3.2. Subsection heading

3.2.1. Sub-subsection heading 


\subsubsection{Sub-subsection heading}

3.2.3. .........

4. Conclusion

5. Acknowledgements

6. References

The total number of pages of this template is usually over 30 . With many subsections included, the structure of the content resembles a direct graph. Meantime, the directed graph works in a one direction only (in terms of sense of an article). In case of an article, it strictly means that the article can only be rightly comprehended in one direction of its reading, "sectionby-section".

The main principles of memory formation say that the associative connection between two objects can be inevitably lost if one of intermediate objects is removed from the connection chain.

In case of the template presented, in order to deeply understand an article written in such template, we strongly need to read the article "section-bysection" spending an extra time for included references.

In becomes a big problem if a reader has a single purpose to get a direct answer for his or her question, "Does the article have a real practical solution?".

\section{Solution}

In light of the fact that the majority of written works is regularly struggling with misconception of its content, it seems crucial to find a way of relevant interpretation of the works.

We propose a first step to finding the way. The following structure describes possible sections of the article that can extremely simplify its comprehension.

Content

1. Introduction

2. Problem

3. Solution

4. Conclusion

Introduction.

This section is as common as the one in the conventional template.

Problem. 
The Problem section can have two main parts, theoretical and practical. The theoretical part describes the problem in terms of probabilities and assumptions. The practical part describes real practical cases that lead to the problem.

\section{Solution.}

As in the Problem section, the Solution section has two parts. The first part describes a theoretical solution while the second one describes a practical solution of practical user cases.

In case if the Problem section has only theoretical part, the article must be considered as a theoretical solution. The same approach is used for the practical part.

\section{Conclusion.}

It is the same as in the conventional template.

In nature of any problem lies a knowledge. The problem is always composed of a group of knowledge [3]. In other words, the problem is a sum of two or more knowledge.

$K_{1} "+" K_{2} "=" P_{3}$, where $K-$ set of knowledge of different kind, $P$ set of problems of different kind.

When we "sum" knowledge $K_{1}$ and $K_{2}$, the result can take two forms, $K_{i}$ (other knowledge) or $P_{i}$ (other problem):

$$
\begin{gathered}
K_{i}+K_{i+1}=K_{i+2} \\
\text { or } \\
K_{i}+K_{i+1}=P_{i+2}
\end{gathered}
$$

from which we have [3]:

$$
\begin{gathered}
K_{i+1}+P_{i+2} \equiv P \\
\text { or } \\
P_{i+2}=\frac{1}{2}\left(P-N_{n}\right)
\end{gathered}
$$




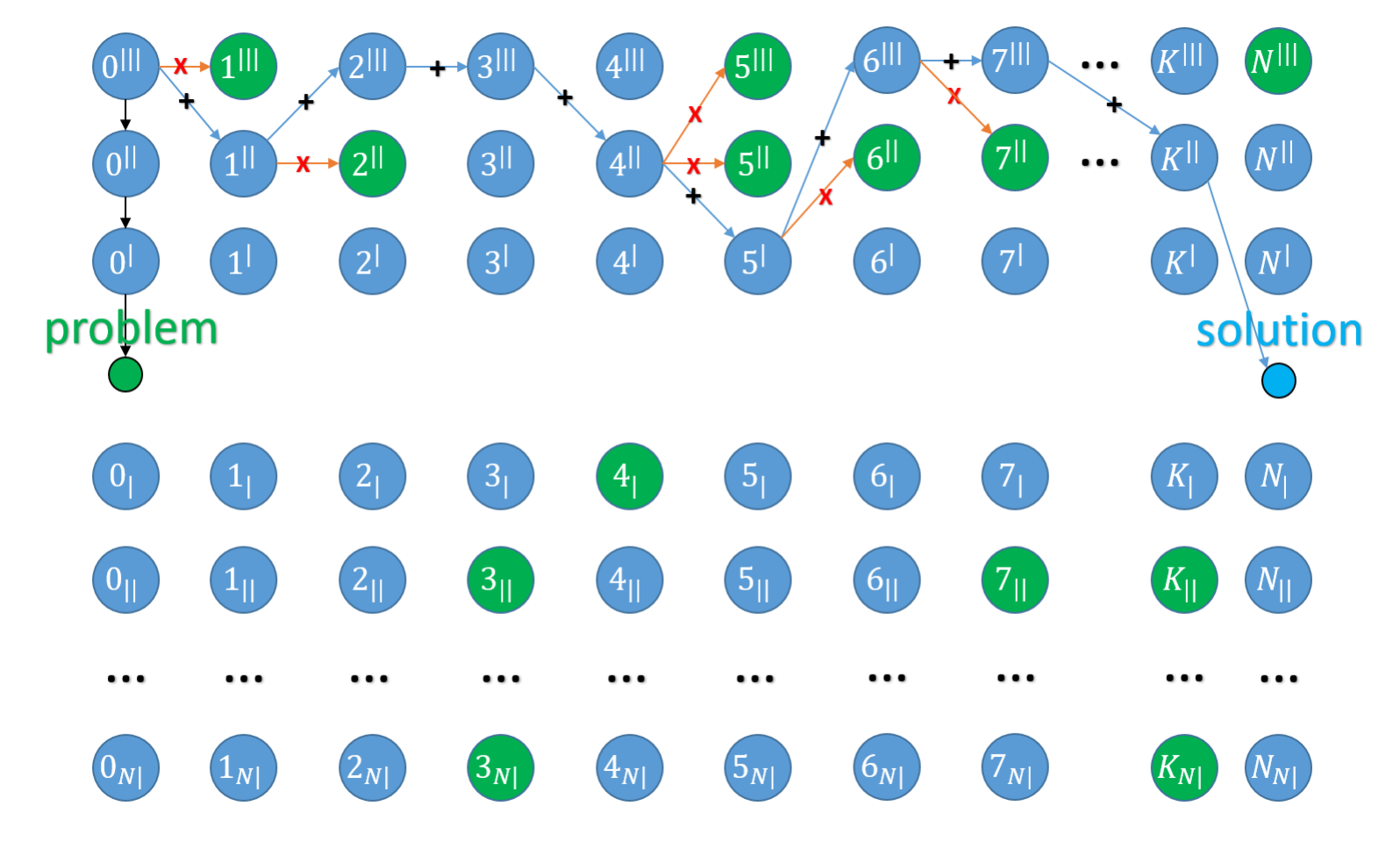

As we see on the figure above, the problem was appeared as a result of treatment (sum) of three knowledge, $0^{\| l l}-0^{\| l}-0^{l}$. The reason of appearance of the problem is that the knowledge, or directly speaking, "symbiosis of knowledge" led to "a pseudo knowledge" (green-colored) that does not have practical application. The pseudo knowledge has only input channel.

The main rule of choosing the right way (blue-colored numbers) to the solution is as follows:

"Deviation of each next-chosen knowledge must be minimum from the row what the very first knowledge is belonged to".

In the case of the last figure, the total "problem-to-solution" way is as $0^{\text {III }}$ $1^{\| l}-2^{\| l \mid}-3^{\| I}-4^{\| \prime}-5^{\mid}-6^{\| I I}-7^{\| I}-K^{\|}$

Sets of $K$ and $P$ are determined as follows:

$$
N<P
$$

Strict inequality is explained by the nature of set of $P$. The set of $P$ has binary operation determined whereas the set of $N$ only unary one. 


\section{Conclusion}

In order to be a standard it should be ruled.

The rules of IPSC standard are as follows:

1. The Problem section should not have any references for other external problem or problems. Instead it should reference to knowledge or a "symbiosis of knowledge" that caused the problem. It should describe a single problem which in turn can consists of other internal problems interconnected with the single one.

2. The Problem section should have theoretical or/and practical sources of its existence.

In other words, it should have a proof of existence of the problem, for example, by referencing to an external resource describing the same problem.

3. The Solution section should not have any references to a solution or solutions of the problem that is not the main (problem section) or internal one. Only if the problem consists of several internal problems, the reference can be used.

4. The Solution section should have theoretical or/and practical description. It should have a clear list of steps for solving the problem. If it uses the practical part, a reference to the external sources is very welcome.

Recommendation for the number of an article's pages as maximum as 7.

As a human neuronet of memory consists of trillions of associative connections, it is extremely reasonable to adhere to a predefined pattern of thinking. The number of the patterns is totally up to a researcher.

We hope that our decent work will help other researchers and innovators in their daily endeavors. 


\section{References}

[1] L. Abbott, "Learning in neural network memories", 1990, http://www.columbia.edu/cu/neurotheory/Larry/AbbottNetwork90.pdf

[2] E. Mielberg, "Decentralized Chain of Transactions", 2018, https://medium.com/@EggerMielberg/neurochain-decentralized-chain-oftransactions-162a31aee001

[3] E. Mielberg, "Theory of One Synapse", to be published, 2019

[4] D. Vogel, "A Neural Network Model Of Memory and Higher Cognitive Functions in the Cerebrum", https://pdfs.semanticscholar.org/3242/35b88af7e63c8662bbe31b0675b5cd fd0af5.pdf

[5] T. Sejnowski, "Memory and Neural Networks", https://papers.cnl.salk.edu/PDFs/Memory\%20and\%20Neural\%20Networks \%201998-3268.pdf

[6] M. Stowe, "Memory Capacity of a Random Neural Network", https://arxiv.org/ftp/arxiv/papers/1211/1211.3451.pdf

[7] B. Widrow, "Cognitive Memory", https://web.stanford.edu/class/ee373b/cognitive memory2.pdf 